\title{
Análisis de los programas de evaluación del desempeño de malaria de los laboratorios de salud pública y privados de colombia 2015-2016
}

\author{
Performance evaluation analysis of malaria's programs of Colombian \\ public and private health laboratories 2015-2016
}

Cortés Cortés Liliana Jazmín ${ }^{1}$, Caicedo Díaz Ricardo Andrés ${ }^{1}$

\section{Resumen}

Introducción. Para el fortalecimiento de la calidad del diagnóstico de malaria en Colombia, se desarrollan los Programas de Evaluación del Desempeńo (PED) en los que participan laboratorios privados y públicos del país. Objetivo. Analizar los resultados obtenidos en los programas de evaluación del desempeño de malaria de los laboratorios de salud pública y privados de Colombia en el lapso 2015-2016. Materiales y métodos. Se realizó un estudio de tipo retrospectivo mediante la revisión de los resultados obtenidos por los LSP y laboratorios privados participantes en los programas de evaluación directa e indirecta del desempeño (PEDD, PEID) de malaria durante los años 2015 y 2016 en términos de participación, concordancia de positividad y negatividad (Índice Kappa de Cohen), concordancia de especie, de formas parasitarias y de recuento (Z score). Resultados. La participación en el PEID se incrementó de 15\% en 2015 a 51\% en 2016, así como el total de láminas enviadas que en su mayoría cumplían con los criterios establecidos por el Laboratorio Nacional de referencia (LNR). La participación en el PEDD se incrementó de $88 \%$ en 2015 a 94\% en 2016, con un Índice Kappa de Cohen de 0,97, una media de concordancia de especie parasitaria de $83,3 \%$ y de formas parasitarias de $62,5 \%$ y una concordancia del recuento parasitario más frecuente entre -0,9 y 0,9, evidenciándose un mejor desempeńo en 2016. Conclusión. Basados en los resultados obtenidos es necesario promover una mayor participación de los LSP en los PED de malaria, especialmente en el PEID y aumentar la participación de los laboratorio privados.

Palabras claves: malaria, calidad, diagnóstico, laboratorio, desempeño, Colombia.

\footnotetext{
1. Grupo de Parasitología. Dirección de Redes en Salud Pública. Instituto Nacional de Salud. Correspondencia: jcortes@ins.gov.co 


\begin{abstract}
Introduction. In order to strengthen the quality of malaria diagnosis in Colombia, Performance Evaluation Programs (PED) are developed in which private and public laboratories of the country participate. Objective. To analyze the results obtained in the malaria evaluation programs of the public and private health laboratories of Colombia from 2015 to 2016. Materials and methods. A retrospective study was carried out by reviewing the results obtained by the LSPs and private laboratories participating in the direct and indirect evaluation programs of malaria during the years 2015 and 2016 in terms of participation, concordance of positivity and negativity (Cohen's Kappa Index), species concordance, parasitic and counting agreement ( $Z$ score). Results. Participation in PEID increased from $15 \%$ in 2015 to $51 \%$ in 2016, as well as the total number of sheets sent, which mostly met the criteria established by the National Reference Laboratory (NRL). Participation in the PEDD increased from $88 \%$ in 2015 to $94 \%$ in 2016, with a Cohen's Kappa Index of 0.97, an average of parasitic species concordance of $83.3 \%$ and parasitic forms of $62.5 \%$ and a more frequent parasitic count concordance between -0.9 and 0.9, showing a better performance in 2016. Conclusion. Based on the results obtained, it is necessary to promote greater participation of LSPs in malaria PEDs, especially in SIDS, and to increase the participation of private laboratories.
\end{abstract}

Keywords: malaria, quality, diagnosis, laboratory, performance, Colombia.

\section{Introducción}

La malaria en Colombia es un problema de salud pública, debido a que las características eco-epidemiológicas del país hacen que el 85\% del territorio rural presente condiciones aptas para la transmisión de la enfermedad y que el $60 \%$ de su población esté en riesgo de contraerla. Además, el sistema de vigilancia presenta una serie de limitaciones en relación al reporte de casos, pues no es lo suficientemente sensible para permitir el análisis inmediato del riesgo de la transmisión (1). De acuerdo con el reporte de casos del Sistema Nacional de Vigilancia en Salud Pública (SIVIGILA), para la semana epidemiológica 52 de 2016, los casos de malaria se concentraron principalmente en los departamentos de Chocó (46.484 casos), Nariño (10.950 casos), Antioquía (7.064 casos), Amazonas (3.536 casos), Guainía (2.463 casos), Córdoba (1.827 casos) y Distrito de Buenaventura (2.850 casos), con un total de 83.356 casos notificados de malaria (2).
Desde el inicio, en el año 1992 de la "Estrategia Global de Control de la Malaria” hasta la iniciativa, en el año 1998 de "Hacer Retroceder la Malaria", se ha hecho énfasis en la importancia de garantizar un diagnóstico temprano y un tratamiento inmediato en el sistema de prestación de servicios de salud, que cuente con un programa de garantía de la calidad para lograr la idoneidad en el diagnóstico de paludismo $(3,4)$; por lo que con el fin de propender por el fortalecimiento continuo de la capacidad y calidad diagnóstica de malaria, el Instituto Nacional de Salud (INS) a través del Laboratorio Nacional de Referencia (LNR) de Parasitología de la Dirección de Redes en Salud Pública (DRSP) organiza los Programas de Evaluación Externa del Desempeño (PEED) y Evaluación Indirecta del Desempeño (PEID) que hacen parte de las actividades misionales (5).

El PEED es un procedimiento estandarizado en el cual se envían láminas de gota gruesa o extendidos 
coloreados con el objetivo de evaluar la idoneidad del diagnóstico de malaria en los laboratorios de la red (6).

Esta estrategia evalúa el desempeño de los laboratorios a partir de un panel de diez (17) láminas anuales (gotas gruesas y extendidos de sangre periférica), que son previamente identificadas individualmente con códigos irrepetibles. Estos ítems están elaborados con especies presentes en la región. Se incluyen láminas con diferentes grados de complejidad en relación a densidad parasitaria, morfología y artefactos; adicionalmente, se envían infecciones mixtas de Plasmodium sp. falciparum (P. falciparum) y Plasmodium vivax (P. vivax) y de otros hemoparásitos, y láminas negativas, todas de calidad óptima. Los parámetros a determinar en los ítems de ensayo son: positividad o negatividad de las muestras, la especie o especies presentes en la muestra, la identificación morfológica de las formas sexuadas y asexuadas y la densidad parasitaria en los ítems de ensayo positivos. Los paquetes son enviados por el LNR de Parasitología a los Laboratorios Departamentales de Salud Pública y privados participantes, para que realicen la lectura y posteriormente, poder evaluar su desempeño. Las láminas son revisadas por un experto antes de su remisión, cumpliendo con las características de homogeneidad y estabilidad óptimas, de tal forma que la complejidad sea similar y que la evaluación sea comparable para todos los participantes (18).

El PEID es una actividad internacionalmente conocida como "seguimiento del desempeńo", que consiste en una evaluación retrospectiva del diagnóstico que se hace de rutina y se aplica a un porcentaje determinado de muestras examinadas inicialmente por los sitios de diagnóstico de malaria, supervisadas por los Laboratorios de Salud Pública (LSP) y finalmente remitidas al Instituto Nacional de Salud (INS) para su revisión y elaboración de informes (19). Los criterios de envío de láminas definidos para esta actividad son: láminas con diagnóstico de especies diferentes a $P$. vivax y $P$. falciparum, láminas con diagnóstico de infección mixta, láminas con re- cuentos parasitarios superiores a 50.000 parásitos/ micro litro (uL) de sangre y láminas de pacientes con malaria complicada y probable mortalidad por malaria. Según la OPS, los programas deben ir del control a la eliminación mediante la reorientación de acciones hacia la eliminación (23).

Dentro de la reorientación de las acciones se señalan algunos cambios dentro del sistema de gestión de la calidad de diagnóstico para que sea completamente funcional (17) y teniendo en cuenta que una de las herramientas para monitorear la calidad del diagnóstico microscópico de malaria son los Programas de Evaluación del Desempeño se realiza el presente análisis de los resultados obtenidos por parte de los LSP y laboratorios privados que participaron durante los ańos 2015-2016.

\section{Materiales y métodos}

Se realizó un estudio de tipo retrospectivo mediante la revisión de los resultados obtenidos por los LSP y laboratorios privados participantes en los programas de evaluación del desempeño de las rondas de los años 2015 y 2016 (7-10).

Se realizó el condensado de los resultados del PEID revisando los registros de ingreso de las láminas enviadas por los diferentes LSP. Se hizo un consolidado y comparación de los resultados obtenidos, en términos de concordancia general (positividad/ negatividad), concordancia de especie parasitaria y recuento parasitario. Teniendo en cuenta que los resultados de estos programas son confidenciales y sólo competen al laboratorio participante, se le asignó un código a cada participante (Tablas 1 y 2).

El PEED de malaria evalúa tres variables de tipo cualitativo nominal: identificación parasitaria (presente/ausente), identificación de especie (P. vivax, $P$. falciparum, P. malariae, P. ovale o infección mixta), identificación de estadios parasitarios (trofozoítos, esquizontes y gametocitos) y una variable cuantitativa la cual es la densidad parasitaria, dada en número de parásitos por micro litro (uL) de sangre. 
Para el análisis de la variable identificación parasitaria se calculó el Índice Kappa de Cohen (k), el cual compara el desempeño del participante, frente al valor asignado del ítem de ensayo, corrigiendo la concordancia debida al azar. Para el análisis de las variables, identificación de especie e identificación de estadio parasitario, se estimaron las frecuencias de concordancia. Finalmente, para el recuento parasitario, se realizó el cálculo del puntaje Z (Z score) (18), el cual estima la distancia en desviaciones estándar entre el resultado de la medición del participante y la media de los resultados de todos los participantes. En este caso, cuando el valor obtenido en la evaluación este más cercano a 0 (cero), más se acerca al valor asignado y mejor será su desempeño (Tabla 3) (19).

\section{Análisis estadístico}

A partir de los consolidados se elaboró una base de datos que incluyó los resultados de las evaluaciones del desempeńo de cada uno de los participantes. De las variables cualitativas se evaluó la concordancia entre observadores, respecto a positividad/ negatividad con el índice Kappa de Cohen, para especie parasitaria y estadios parasitarios, se estima la frecuencia de concordancia. Para la variable de los Z score, se analizó la frecuencia del puntaje por medio de rangos. Los datos fueron tabulados y procesados en hojas de cálculo (Excel Vista ${ }^{\circledR}$ ).

\section{Resultados}

En relación al Programa de Evaluación Indirecta del Desempeño, se evidenció que en el año 2015 solo el 15\% (5/33) de los laboratorios departamentales reportaron esta actividad. Sin embargo, para el año 2016 el porcentaje de participación se incrementó a un 51\% (17/33).

Sobre el total de láminas enviadas en las dos rondas del PEID, se evidencia que en su mayoría cumplían con los criterios establecidos. Se recibieron láminas de infecciones mixtas, recuentos superiores de 50.000 parásitos/uL de sangre y láminas de posibles mortalidades por malaria. Se evidenció un caso de malaria por Plasmodium ovale, y ninguna lámina de $P$. malariae (Tabla 4).

Para 2015, el total de láminas enviadas fue de 7 con un promedio de concordancia general en identificación parasitaria (positivo/negativos) del $100 \%$, y concordancia en identificación de especie del 100\% (Tabla 1).

En el año 2016 se enviaron en esta actividad un total de 69 láminas. El promedio de concordancia en identificación parasitaria fue $100 \%$, el percentil 90 de la identificación de especie fue 100\% (Tabla 2).

Respecto a la concordancia de recuento, tanto para las láminas enviadas durante el 2015 como el 2016, todas cumplieron con el criterio de la Organización Panamericana de la Salud (OPS) respecto a una concordancia $\geq 50 \%$ con respecto al recuento reportado por el Laboratorio Nacional de Referencia.

Para el PEED de malaria, durante el año 2015 participaron 38 laboratorios (29 LSP (88\%) y 9 laboratorios privados), obteniéndose un promedio del Índice Kappa de Cohen de 0,97 respecto al ítem de positividad/negatividad. Para el año 2016 participaron 40 laboratorios (31 LSP (94\%) y 9 laboratorios privados), obteniéndose también un promedio de Kappa de 0,97 respecto al ítem de positividad/negatividad. Los porcentajes de concordancia con relación a la especie parasitaria se calcularon por cada una de las muestras evaluadas en cada uno de los ańos, al igual que el porcentaje de concordancia por estadio parasitario. (Tabla 5 y 6).

Con relación a la evaluación de la concordancia en la variable cuantitativa, el recuento parasitario se midió en " $Z$ score". Los resultados más frecuentes fueron entre $-0,9$ y 0,9 , en ambas rondas. Sin embargo, se evidencia un mejor desempeño en 2016 (Tabla 3). 


\section{Discusión}

Es necesario fortalecer la participación de los Laboratorios de Salud Pública y Secretarías de Salud en el programa de PEID de malaria (20), ya que aunque el objetivo de ajustar los criterios de envío de las láminas de malaria fue disminuir el número de láminas enviadas al LNR, todos los departamentos deben participar en esta actividad como parte del aseguramiento de la calidad del diagnóstico. Además, teniendo en cuenta el número de casos de infecciones mixtas y de complicaciones y mortalidades por malaria notificados al SIVIGILA durante estos años, no se está cumpliendo con los criterios de envío definidos por el LNR para este evento.

Los resultados obtenidos por los laboratorios participantes son una herramienta importante para monitorear la calidad del diagnóstico (21), especialmente bajo criterios que no son de rutina diagnóstica y que implican un grado mayor de habilidad y conocimiento por parte del microscopista. Aunque los resultados obtenidos muestran en general buenas concordancias de especie y recuento, es de anotar que hubo laboratorios que tuvieron concordancias muy bajas, lo que permite identificar falencias para que los LSP programen readiestramientos para el talento humano de la red de diagnóstico de malaria departamental y municipal, tanto en el ámbito público como en el privado (22).

Por otra parte, ya que el número de láminas recibidas en el LNR del INS para esta actividad es menor, esto permite que los resultados de esta actividad sean oportunos y permitan impactar directamente en la salud del paciente si hubo errores de diagnóstico que conllevaron a la administración de un tratamiento inadecuado.

La participación en el PEED de malaria por parte de los LSP fue mayor al $85 \%$, porcentaje que aunque es bueno, se debe incrementar ya que esta actividad debe contar con la participación del 100\% de los LSP del país, siendo uno de los principales obstáculos la estabilidad de recurso humano (8).

Respecto al porcentaje de acuerdo PEED durante el año 2015, el menor porcentaje de concordancia de especie $(72,2 \%)$ y de estadios parasitarios (50\%) se obtuvo con la muestra 3, la cual era positiva para $P$. vivax, con un alto porcentaje de trofozoitos jóvenes, lo que generó confusión en muchos participantes que la diagnosticaron erróneamente como $P$. falciparum o infección mixta y que solamente vieron las formas de trofozoitos y no evidenciaron la presencia de esquizontes ni gametocitos en la muestra (13-15).

Respecto al porcentaje de acuerdo PEED durante el año 2016, el menor porcentaje de concordancia de especie (75\%) se obtuvo con la muestra 7, la cual era positiva para $P$. vivax, con una baja parasitemia, lo que generó confusión en muchos participantes que la diagnosticaron erróneamente como P. falciparum o P. malariae. En cuanto a estadios parasitarios el menor porcentaje de concordancia se obtuvo en la muestra $4(20 \%)$ ya que solamente vieron las formas de trofozoitos y no evidenciaron la presencia de esquizontes ni gametocitos en la muestra. En estudios realizados en Perú y Venezuela, en donde se evaluó la calidad del diagnóstico hecho por los microscopistas, se hallaron concordancias entre $50 \%$ y $70 \%$ encontrándose que los factores que afectan la concordancia son la falta de actualización y el tiempo de permanencia de los microscopistas en el sitio de diagnóstico (23). Estos hallazgos también se pueden aplicar a las bajas concordancias en algunas de las muestras examinadas en el PEED de nuestro país (24-26).

\section{Agradecimientos}

Los autores expresan su agradecimiento a los Laboratorios de Salud Pública y Secretarías de Salud y a los laboratorios privados participantes en las actividades de PEID y PEED de malaria. 


\section{Declaración de conflicto de intereses}

Los autores declaran que durante las etapas de recolección de la información y análisis de los datos, no incurrieron en conflictos de intereses que hubieran podido afectar los resultados.

\section{Financiación}

Los recursos económicos necesarios para el desarrollo del presente estudio fueron aportados por el Instituto Nacional de Salud, Dirección de Redes en Salud Pública, Subdirección Laboratorio Nacional de Referencia, Grupo de Parasitología 2017.

\section{Referencias}

1. Vallejo A, Chaparro P, Benavides Y, Álvarez A, Quintero J, Padilla J, et al. High prevalence of sub-microscopic infections in Colombia. Malaria J. 2015; 14 (201):1-7, https://doi. org/10.1186/s12936-015-0711-6.

2. Instituto Nacional de Salud. Protocolo para la vigilancia en salud pública de malaria. http://www.ins.gov.co/lineas-de-accion/Subdireccion-Vigilancia/sivigila/Protocolos\%20SIVIGILA/PRO\%20Malaria.pdf. Bogotá: Instituto Nacional de Salud, 2017.

3. Ministerio de Salud. Implementación de la iniciativa de hacer retroceder el paludismo en Colombia. Semana Epidemiológica No. 37. Bogotá: Ministerio de Salud; 2000.

4. World Health Organization. Technical consultation to update the who malaria microscopy quality assurance manual: meeting report. Geneva: World Health Organization; 2014.

5. Ministerio de la Protección Social. Decreto 2323 del 13 de julio de 2006. Diario oficial 2006; 46:328. Bogotá: Ministerio de la Protección Social; 2006.

6. Organismo Andino de Salud, PAMAFRO. Propuesta para el fortalecimiento de la gestión de calidad del diagnóstico microscópico a nivel nacional y a nivel departamental (áreas priorizadas en Colombia durante la fase II). Bogotá: OAS; 2008.

7. Bernal Luz Mery, López Greizy. Diagnóstico prénatal: retrospectiva. Nova. 2014; 12( 21 ): 23-36.

8. Márquez Gómez Marco Antonio, Gómez Díaz Graciela María. Accidente ofídico en el departamento de Sucre, Colombia. Nova. 2015; 13( 24 ): 39-46.

9. Corrales Lucia Constanza, Antolinez Romero Diana Marcela,
Bohórquez Macías Johanna Azucena, Corredor Vargas Aura Marcela. Bacterias anaerobias: procesos que realizan y contribuyen a la sostenibilidad de la vida en el planeta. Nova. 2015; 13( 24 ): 55-81.

10. González Yuri Lilian. Evaluación de la percepción del riesgo ocupacional en trabajadores de una empresa del sector de la construcción en Bogotá D.C. Nova. 2015; 13( 23 ): 93-107.

11. Mendoza NM, González NE. Performance assessment employing slide sets: A tool for the classification of senior microscopists from Colombia's Malaria Control Program. Biomédica. 2015; 35(4):582-9. https://doi.org/10.7705/biomedica. v35i4.2694

12. Ospina OL, Cortés LJ, Cucunubá ZM, Mendoza NM, Chaparro P. Caracterización de la Red Nacional de Diagnóstico de Malaria, Colombia, 2006-2010. Biomédica. 2012; 32: 46-57. https://doi.org/10.7705/biomedica.v32i0.584

13. Carrero Sandra Helena Suescún, HerediaMontoya Dina Paola, Bolaños Yoryany Mulato, Medellín Martín Orlando Pulido. Seroprevalencia de infección por Leptospira y factores de riesgo en estudiantes de una universidad de Colombia. Nova. 2017; 15 ( 27 ): 131-138.

14. Naranjo Flórez Ricardo Andrés. Avances y perspectivas en Síndrome de Asperger. Nova. 2014; 12( 21 ): 81-101.

15. Zuluaga Martha, Robledo Sebastian, Osorio-Zuluaga German A, Yathe Laura, Gonzalez Diana, Taborda Gonzalo. Metabolomics and pesticides: systematic literature review using graph theory for analysis of references. Nova. 2016; 14( 25 ): 121138.

16. Organización Panamericana de la Salud. Guía para la reorientación de los programas de control de la malaria con miras a la eliminación. Disponible en http://www.usaidami.com/extras/ Guia_para_la_reorientacion_control_malaria_revisada.pdf Ginebra: OPS; 2011.

17. Blair S. Retos para la eliminación de la malaria en Colombia: un problema de saber o de poder. Biomédica. 2012; 32 suppl.1

18. Fryar CD, Gu Q, Ogden CL. Anthropometric reference data for children and adults: United States, 2007-2010. National Center for Health Statistics. Vital Health Stat 11 (252).2012. http://www.statisticshowto.com/probability-and-statistics/z-score.

19. Frean J, Perovic O, Fensham V, McCarthy K, Gottberg A, de Gouveia L, et al. External quality assessment of national public health laboratories in Africa, 2002-2009. Bull World Health Organ. 2012; 90(3):191-199A. https://doi:10.2471/ BLT.11.091876.

20. Carter JY. External quality assessment in resource-limited countries. Biochemia Médica. 2017; 27(1):97-109. https:// doi:10.11613/BM.2017.013.

21. World Health Organization. Technical consultation to update the WHO malaria microscopy quality assurance manual: meeting report. Geneva: World Health Organization; 2014.

22. Odhiambo F, Buff AM, Moranga C, Moseti CM, Wesongah 
JO, Lowther SA, et al. Factors associated with malaria microscopy diagnostic performance following a pilot quality-assurance programme in health facilities in malaria low-transmission areas of Kenya, 2014. Malar J. 2017; 16(1):371. https:// doi:10.1186/s12936-017-2018-2

23. Rosas-Aguirre A, Gamboa D, Rodríguez H, Llanos-Zavalaga F, Aguirre K, Llanos-Cuentas A. Uso de paneles de láminas estandarizadas para la evaluación de competencias en el diagnóstico microscópico de la malaria en la Amazonía Peruana. Rev Perú Med Exp Salud Pública. 2010; 27(4): 540-47.

24. Ávila de Navia Sara Lilia, Estupiñán-Torres Sandra Mónica, Díaz González Liliana. Calidad bacteriológica del agua Vereda El Charco, San Miguel de Sema, Boyacá- Colombia. Nova. 2016; 14( 25 ): 139-145.

25. Almonacid Urrego Carmen Cecilia, Camarillo Romero María del Socorro, Gil Murcia Zulay, Medina Medina Claudia Yasmin, Rebellón Marulanda Jennifer Viviana, Mendieta Zerón Hugo. Evaluación de factores de riesgo asociados a enfermedad cardiovascular en jóvenes universitarios de la Localidad Santafé en Bogotá, Colombia. Nova. 2016; 14(25): 9-17.

26. González Devia Johanna L., Monroy Romero Paola A., Almonacid Urrego Carmen C.. Homocisteína y otros factores de riesgo cardiovascular en niños de educación básica primaria del Colegio Distrital Manuel Elkin Patarroyo, Bogotá, D.C. Colombia. Estudio piloto. Nova. 2017 ; 15( 27 ): 103-117. 


\section{Anexos}

Tabla 1. Resultados de PEID Malaria 2015.

\begin{tabular}{|l|l|l|l|}
\multicolumn{5}{c}{ Programa de Evaluación Indirecta del Desempeńo Malaria 2015 } \\
\hline $\begin{array}{c}\text { Laboratorio de Salud } \\
\text { Pública }\end{array}$ & $\begin{array}{c}\text { Láminas enviadas según } \\
\text { criterios establecidos }\end{array}$ & $\begin{array}{c}\text { Concordancia en } \\
\text { identificación parasitaria }\end{array}$ & $\begin{array}{c}\text { Concordancia en } \\
\text { identificación de especie }\end{array}$ \\
\hline 1 & 1 & 100 & 100 \\
\hline 2 & 1 & 100 & 100 \\
\hline 3 & 1 & 100 & 100 \\
\hline 4 & 3 & 100 & 100 \\
\hline 5 & 1 & 100 & 100 \\
\hline Total & 7 & NA & NA \\
\hline
\end{tabular}

Fuente. Elaboración propia.

Tabla 2. Resultados de PEID Malaria 2016.

\begin{tabular}{|l|l|l|l|}
\hline \multicolumn{5}{c}{ Programa de Evaluación Indirecta del Desempeńo Malaria 2016 } \\
\hline $\begin{array}{c}\text { Laboratorio de Salud } \\
\text { Pública }\end{array}$ & $\begin{array}{c}\text { Láminas enviadas según } \\
\text { criterios establecidos }\end{array}$ & $\begin{array}{c}\text { Concordancia en } \\
\text { identificación parasitaria }\end{array}$ & $\begin{array}{c}\text { Concordancia en } \\
\text { identificación de especie }\end{array}$ \\
\hline 6 & 13 & 100 & 100 \\
\hline 7 & 8 & 100 & 100 \\
\hline 8 & 2 & 100 & 100 \\
\hline 9 & 4 & 100 & 100 \\
\hline 10 & 3 & 100 & 0 \\
\hline 11 & 6 & 100 & 100 \\
\hline 12 & 2 & 100 & 100 \\
\hline 13 & 2 & 100 & 100 \\
\hline 14 & 1 & 100 & 100 \\
\hline 15 & 3 & 100 & 0 \\
\hline 16 & 6 & 100 & 100 \\
\hline 17 & 4 & 100 & 100 \\
\hline 18 & 4 & 100 & 100 \\
\hline 19 & 1 & 100 & 50 \\
\hline 20 & 2 & 100 & 100 \\
\hline 21 & 6 & 100 & 83 \\
\hline 22 & 2 & 100 & 100 \\
\hline Total & 69 & NA & NA \\
\hline & & & \\
\hline
\end{tabular}

Fuente. Elaboración propia. 
Tabla 3. Distribución de Z Score según año.

\begin{tabular}{|l|l|l|l|l|}
\hline \multicolumn{1}{|c|}{ Rango } & \multicolumn{1}{c|}{ Frecuencia } & \multicolumn{2}{c|}{2016} \\
\hline$-4,0$ a $-2,0$ & 2 & $1,5 \%$ & 0 & \multicolumn{2}{c|}{ Frecuencia } \\
\hline$-1,99$ a $-1,00$ & 17 & $12,6 \%$ & 13 & $0,0 \%$ \\
\hline$-0,99$ a 0,99 & 99 & $73,3 \%$ & 191 & $5,8 \%$ \\
\hline 1,00 a 1,99 & 15 & $11,1 \%$ & 12 & $85,7 \%$ \\
\hline 2,0 a 3,0 & 1 & $0,7 \%$ & 2 & $5,4 \%$ \\
\hline Mayor a 3,0 & 1 & $0,7 \%$ & 5 & $0,9 \%$ \\
\hline Total & 135 & $100,0 \%$ & 223 & $2,2 \%$ \\
\hline
\end{tabular}

Fuente. Elaboración propia.

Tabla 4. Número de láminas para PEID según criterio.

\begin{tabular}{|l|l|l|}
\hline \multicolumn{1}{|c|}{ Criterio } & \multicolumn{1}{c|}{2015} \\
\hline $\begin{array}{l}\text { Infección por Plasmodium falciparum (recuentos mayores a 50.000 parasitos/ } \\
\text { uL, malaria complicada o mortalidad) }\end{array}$ & 2 & 32 \\
\hline $\begin{array}{l}\text { Infección por Plasmodium vivax (recuentos mayores a 50.000parasitos/uL, } \\
\text { malaria complicada o mortalidad) }\end{array}$ & 3 & 20 \\
\hline Infección mixta & 2 & 4 \\
\hline Laminas negativas & 0 & 12 \\
\hline Infección por Plasmodium ovale & 0 & 1 \\
\hline Total & 7 & 69 \\
\hline
\end{tabular}

Fuente. Elaboración propia.

Tabla 5. Porcentaje de acuerdo PEED 2015 en evaluación de concordancia de especie y estado según muestras positivas.

\begin{tabular}{|l|c|l|}
\hline \multicolumn{3}{|c|}{ Porcentaje de acuerdo PEED 2015 } \\
\hline Muestra & Especie & Estadios parasitarios \\
\hline 1 & $100,0 \%$ & $72,2 \%$ \\
\hline 3 & $72,2 \%$ & $50,0 \%$ \\
\hline 7 & $77,8 \%$ & $50,0 \%$ \\
\hline 9 & $83,3 \%$ & $77,8 \%$ \\
\hline Media & $83,3 \%$ & $62,5 \%$ \\
\hline
\end{tabular}

Tabla 6. Porcentaje de acuerdo PEED 2016 en evaluación de concordancia de especie y estado según muestras positivas

Fuente. Elaboración propia.

\begin{tabular}{|l|c|l|}
\hline \multicolumn{3}{|c|}{ Porcentaje de acuerdo PEED 2016} \\
\hline Muestra & Especie & \multicolumn{1}{|c|}{ Estadios parasitarios } \\
\hline 2 & $92,5 \%$ & $67,5 \%$ \\
\hline 3 & $92,5 \%$ & $85,0 \%$ \\
\hline 4 & $87,5 \%$ & $20,0 \%$ \\
\hline 6 & $95,0 \%$ & $80,0 \%$ \\
\hline 7 & $75,0 \%$ & $30,0 \%$ \\
\hline 10 & $85,0 \%$ & $32,5 \%$ \\
\hline Media & $87,9 \%$ & $52,5 \%$ \\
\hline
\end{tabular}

Fuente. Elaboración propia. 